\title{
Four-dimensional black holes with scalar hair in nonlinear electrodynamics
}

\author{
José Barrientos ${ }^{1,2, a}$, P. A. González ${ }^{3, b}$, Yerko Vásquez ${ }^{4, c}$ \\ ${ }^{1}$ Departamento de Física, Universidad de Concepción, Casilla 160-C, Concepción, Chile \\ ${ }^{2}$ Departamento de Enseñanza de las Ciencias Básicas, Universidad Católica del Norte, Larrondo 1281, Coquimbo, Chile \\ ${ }^{3}$ Facultad de Ingeniería, Universidad Diego Portales, Avenida Ejército Libertador 441, Casilla 298-V, Santiago, Chile \\ ${ }^{4}$ Departamento de Física y Astronomía, Facultad de Ciencias, Universidad de La Serena, Avenida Cisternas 1200, La Serena, Chile
}

Received: 14 June 2016 / Accepted: 21 November 2016 / Published online: 7 December 2016

(c) The Author(s) 2016. This article is published with open access at Springerlink.com

\begin{abstract}
We consider a gravitating system consisting of a scalar field minimally coupled to gravity with a selfinteracting potential and a U(1) nonlinear electromagnetic field. Solving analytically and numerically the coupled system for both power-law and Born-Infeld type electrodynamics, we find charged hairy black hole solutions. Then we study the thermodynamics of these solutions and we find that at a low temperature the topological charged black hole with scalar hair is thermodynamically preferred, whereas the topological charged black hole without scalar hair is thermodynamically preferred at a high temperature for power-law electrodynamics. Interestingly enough, these phase transitions occur at a fixed critical temperature and do not depend on the exponent $p$ of the nonlinear electrodynamics.
\end{abstract}

\section{Contents}

1 Introduction ................ 1

2 Four-dimensional black holes with scalar hair in nonlinear electrodynamics . . . . . . . . . . . . . 2

2.1 Power-law electrodynamics . . . . . . . . . . 3

2.2 Born-Infeld type electrodynamics . . . . . . 7

3 Thermodynamics .................. . . 9

4 Conclusions . . . . . . . . . . . . . 11

Appendix A: Analytical solutions . . . . . . . . . . . . . . 12

References . . . . . . . . . . . . . . . 13

\footnotetext{
a e-mail: josebarrientos@udec.cl

b e-mail: pablo.gonzalez@udp.cl

c e-mail: yvasquez@userena.cl
}

\section{Introduction}

Hairy black holes are interesting solutions to Einstein's theory of gravity and also to certain types of modified gravity theories. The first attempts to couple a scalar field to gravity was done in an asymptotically flat spacetime finding hairy black hole solutions [1-3], but it was realized that these solutions were not physically acceptable as the scalar field was divergent on the horizon and stability analysis showed that they were unstable [4]. Also, asymptotically flat black holes with scalar field minimally coupled to gravity were found in $[5,6]$, which evade the no hair theorems by allowing partially negative self-interacting potential, which is in conflict with the dominant energy condition. Some of these solutions were found to be stable for some parameter range [6]. On the other hand, by introducing a cosmological constant, hairy black hole solutions with a minimally coupled scalar field and a self-interaction potential in asymptotically dS space were found, but unstable [7,8]. Also, a hairy black hole configuration was reported for a scalar field non-minimally coupled to gravity [9], but a perturbation analysis showed the instability of the solution $[10,11]$. In the case of a negative cosmological constant, stable solutions were found numerically for spherical geometries $[12,13]$ and an exact solution in asymptotically AdS space with hyperbolic geometry was presented in [14]. A study of general properties of black holes with scalar hair with spherical symmetry can be found in Ref. [15]. Further hairy solutions were reported in [16-24] with various properties. Furthermore, charged hairy solutions were also found, for instance in [25], a topological black hole dressed with a conformally coupled scalar field and electric charge was studied. An electrically charged black hole solution with a scalar field minimally coupled to gravity and electromagnetism was presented in [26]. Recently, for a gravitating system consisting of a scalar field mini- 
mally coupled to gravity with a self-interacting potential and $\mathrm{U}(1)$ electromagnetic field, exact charged hairy black hole solutions with the scalar field which is regular outside the event horizon have been found in [27-29]. Also, new hairy black hole solutions, boson stars and numerical rotating hairy black hole solutions were discussed [30-35], as well as timedependent hairy black holes $[36,37]$. For a review of hairy black holes we refer the reader to [38].

In this work, we extend our previous work $[20,27]$ and we consider a gravitating system consisting of a scalar field minimally coupled to gravity with a self-interacting potential and U(1) nonlinear electromagnetic field. Then we obtain black hole solutions for power-law and Born-Infeld type electrodynamics and we study the thermodynamics and the phase transitions between hairy charged black holes dressed with a scalar field and no hairy charged black holes, focusing on the effects of the nonlinearity of the Maxwell source. The interest in nonlinear electrodynamics arises with the order to eliminate the problem of the infinite energy of the electron by Born and Infeld [39]. Also, nonlinear electrodynamics emerges in the modern context of the low-energy limit of heterotic string theory [40-42], and it plays an important role in the construction of regular black hole solutions [43-48]. Some black holes/branes solutions in a nonlinear electromagnetic field have been investigated for instance in [49-56] and references therein. The thermodynamics of Einstein-Born-Infeld black holes with a negative cosmological constant was studied in [57] and for a power-law electrodynamic in [58], where the authors showed that a set of small black holes are locally stable by computing the heat capacity and the electrical permittivity. The thermodynamics of Gauss-Bonnet black holes for a power-law electrodynamic was studied in [59]. On the other hand, higher dimensional black hole solutions to Einstein-dilaton theory coupled to Maxwell field were found in $[60,61]$ and black hole solutions to Einstein-dilaton theory coupled to Born-Infeld and power-law electrodynamics were found in $[62,63]$.

The phase transitions have been of great interest since the discovery of a phase transition by Hawking and Page in a four-dimensional Schwarzschild AdS background [64]. Witten [65] has extended this four-dimensional transition to arbitrary dimension and provided a natural explanation of a confinement/deconfinement transition on the boundary field theory via the AdS/CFT correspondence. However, phase transitions have recently garnered a great deal of attention motivated mainly by the relationship between the phase transitions and holographic superconductivity $[66,67]$ in the context of the AdS/CFT correspondence. Furthermore, the effects of nonlinear electrodynamics on the properties of the holographic superconductors have recently been investigated [68-77]. It is well known that these phase transitions can be obtained by considering black holes as states in a same grand canonical ensemble and by comparing the free energy associated with each of them. Therefore, it is necessary to find the conserved charge of the theory. It is worth mentioning that the phase-transition phenomena have been analyzed and classified by exploiting Ehrenfest's scheme [7881]. Another point of view to study phase transitions is to consider Bragg-Williams' construction of a free energy function [82]. Also, it was shown that if the space is flat, then the Reissner-Nordström black hole is thermodynamically preferred, whereas if the space is AdS the hairy charged black hole is thermodynamically preferred at a low temperature [27].

The work is organized as follows. In Sect. 2 we present the general formalism. Then we derive the field equations and we find hairy black hole solutions. In Sect. 3 we study the thermodynamics of our solutions and in Sect. 4 we present our conclusions.

\section{Four-dimensional black holes with scalar hair in nonlinear electrodynamics}

The four-dimensional Einstein-Hilbert action with a scalar field minimally coupled to curvature having a self-interacting potential $V(\phi)$ in the presence of a nonlinear electromagnetic field is

$I=\int \mathrm{d}^{4} x \sqrt{-g}\left(\frac{1}{2 \kappa} R+\mathcal{L}\left(F^{2}\right)-\frac{1}{2} g^{\mu \nu} \nabla_{\mu} \phi \nabla_{\nu} \phi-V(\phi)\right)$,

where $\kappa=8 \pi G$, with $G$ the Newton constant and $\mathcal{L}\left(F^{2}\right)$ an arbitrary function of the electromagnetic invariant $F^{2}=$ $F_{\alpha \beta} F^{\alpha \beta}$. The resulting field equations from the above action are

$R_{\mu \nu}-\frac{1}{2} g_{\mu \nu} R=\kappa\left(T_{\mu \nu}^{(\phi)}+T_{\mu \nu}^{(F)}\right)$,

where the energy-momentum tensors $T_{\mu \nu}^{(\phi)}$ and $T_{\mu \nu}^{(F)}$ for the scalar and electromagnetic fields are

$$
\begin{aligned}
T_{\mu \nu}^{(\phi)} & =\nabla_{\mu} \phi \nabla_{\nu} \phi-g_{\mu \nu}\left[\frac{1}{2} g^{\rho \sigma} \nabla_{\rho} \phi \nabla_{\sigma} \phi+V(\phi)\right], \\
T_{\mu \nu}^{(F)} & =g_{\mu \nu} \mathcal{L}\left(F^{2}\right)-4 \frac{\mathrm{d} \mathcal{L}\left(F^{2}\right)}{\mathrm{d} F^{2}} F_{\mu}{ }^{\lambda} F_{\nu \lambda},
\end{aligned}
$$

respectively. Using Eqs. (2) and (3) we obtain the equivalent equation

$$
\begin{aligned}
& R_{\mu \nu}-\kappa\left(\partial_{\mu} \phi \partial_{\nu} \phi+g_{\mu \nu} V(\phi)\right)=\kappa\left(-4 \frac{\mathrm{d} \mathcal{L}\left(F^{2}\right)}{\mathrm{d} F^{2}} F_{\mu}{ }^{\alpha} F_{\nu \alpha}\right. \\
& \left.+2 g_{\mu \nu} F^{2} \frac{\mathrm{d} \mathcal{L}\left(F^{2}\right)}{\mathrm{d} F^{2}}-g_{\mu \nu} \mathcal{L}\left(F^{2}\right)\right) .
\end{aligned}
$$

Now, if we consider the following metric ansatz:

$$
\mathrm{d} s^{2}=-f(r) \mathrm{d} t^{2}+f^{-1}(r) \mathrm{d} r^{2}+a^{2}(r) \mathrm{d} \Omega^{2},
$$


where $\mathrm{d} \Omega^{2}$ is the metric of the spatial 2 section, which can have a positive, negative or zero curvature, and $A_{\mu}=$ $\left(A_{t}(r), 0,0,0\right)$ is the scalar potential of the electromagnetic field. We find the following three independent differential equations:

$$
\begin{aligned}
& f^{\prime \prime}(r)+2 \frac{a^{\prime}(r)}{a(r)} f^{\prime}(r)+2 \kappa V(\phi)=2 \kappa \mathcal{L}\left(F^{2}\right), \\
& f^{\prime \prime}(r)+2 \frac{a^{\prime}(r)}{a(r)} f^{\prime}(r)+\left(4 \frac{a^{\prime \prime}(r)}{a(r)}+2 \kappa \phi^{\prime}(r)^{2}\right) f(r) \\
& +2 \kappa V(\phi)=2 \kappa \mathcal{L}\left(F^{2}\right), \\
& \frac{a^{\prime}(r)}{a(r)} f^{\prime}(r)+\left(\left(\frac{a^{\prime}(r)}{a(r)}\right)^{2}+\frac{a^{\prime \prime}(r)}{a(r)}\right) f(r)-\frac{k}{a(r)^{2}}+\kappa V(\phi) \\
& =\kappa \mathcal{L}\left(F^{2}\right)+4 \kappa A_{t}^{\prime}(r)^{2} \frac{\mathrm{d} \mathcal{L}\left(F^{2}\right)}{\mathrm{d} F^{2}},
\end{aligned}
$$

where $k=1,0,-1$ parameterizes the curvature of the spatial 2 -section. So, if we eliminate the potential $V(\phi)$ from the above equations we obtain

$$
\begin{aligned}
& a^{\prime \prime}(r)+\frac{1}{2} \kappa \phi^{\prime}(r)^{2} a(r)=0, \\
& f^{\prime \prime}-2\left(\left(\frac{a^{\prime}(r)}{a(r)}\right)^{2}+\frac{a^{\prime \prime}(r)}{a(r)}\right) f(r)+\frac{2 k}{a(r)^{2}} \\
& =-8 \kappa A_{t}^{\prime}(r)^{2} \frac{\mathrm{d} \mathcal{L}\left(F^{2}\right)}{\mathrm{d} F^{2}} .
\end{aligned}
$$

In the following, we will work in units where $\kappa=1$. By considering the scalar field studied in [27],

$\phi(r)=\frac{1}{\sqrt{2}} \ln \left(1+\frac{v}{r}\right)$,

where $v$ is a parameter controlling the behavior of the scalar field and it has the dimension of length, from Eqs. (9) and (11) we determine the function $a(r)$, which reads

$a(r)=\sqrt{r(r+v)}$.

Also, from the Maxwell equations

$\partial_{\mu}\left(\sqrt{-g} F^{\mu \nu} \frac{\mathrm{d} \mathcal{L}\left(F^{2}\right)}{\mathrm{d} F^{2}}\right)=0$,

we obtain the following relation:

$A_{t}^{\prime}(r) \frac{\mathrm{d} \mathcal{L}\left(F^{2}\right)}{\mathrm{d} F^{2}}=-\frac{\tilde{Q}}{a(r)^{2}}$,

where $\tilde{Q}$ is an integration constant. We can also determine the metric function $f(r)$ replacing (12) and (14) in (10)

$$
\begin{aligned}
f(r)= & -\frac{\Lambda}{3} r^{2}-\frac{1}{3} v\left(6 \alpha_{2}+\Lambda\right) r+k-\alpha_{2} v^{2} \\
& -2 \alpha_{2} r(r+v) \ln \left(\frac{r}{r+v}\right) \\
& -8 r(r+v) \int \frac{\int r(r+v) A_{t}^{\prime}(r)^{2} \frac{\mathrm{d} \mathcal{L}\left(F^{2}\right)}{\mathrm{d} F^{2}} \mathrm{~d} r}{r^{2}(r+v)^{2}} \mathrm{~d} r
\end{aligned}
$$

$$
\begin{aligned}
= & -\frac{\Lambda}{3} r^{2}-\frac{1}{3} v\left(6 \alpha_{2}+\Lambda\right) r+k-\alpha_{2} v^{2} \\
& -2 \alpha_{2} r(r+v) \ln \left(\frac{r}{r+v}\right) \\
& +8 \tilde{Q} r(r+v) \int_{\infty}^{r} \frac{A_{t}(r)}{r^{2}(r+v)^{2}} \mathrm{~d} r .
\end{aligned}
$$

To find hairy black hole solutions the differential equations have to be supplemented with the Klein-Gordon equation of the scalar field, which in general coordinates reads

$\square \phi=\frac{\mathrm{d} V}{\mathrm{~d} \phi}$,

and whose solution for the self-interacting potential $V(r)$ is

$V(r)=\frac{v^{2}}{2} \int \frac{f^{\prime}(r)}{r^{2}(r+v)^{2}} \mathrm{~d} r$.

In the following sections we will consider two specific electromagnetic Lagrangians $\mathcal{L}\left(F^{2}\right)$ : power-law and Born-Infeld type electrodynamics.

\subsection{Power-law electrodynamics}

In this section we consider power-law electrodynamics characterized by the following Lagrangians [83]:

$\mathcal{L}\left(F^{2}\right)=\eta\left|F^{2}\right|^{p}$,

where $p$ is a rational number and the absolute value ensures that any configuration of electric and magnetic fields can be described by these Lagrangians. One could also consider the Lagrangian without the absolute value and the exponent $p$ restricted to being an integer or a rational number with an odd denominator [84]. The sign of the coupling constant $\eta$ will be chosen such that the energy density of the electromagnetic field is positive; that is, the minus $T_{t}^{t(F)}$ component of the electromagnetic energy-momentum tensor must be positive,

$-T_{t}^{t(F)}=\eta\left|F^{2}\right|^{p}(2 p-1)>0$.

This condition is guaranteed in the following cases: $p>1 / 2$ and $\eta>0$ or $p<1 / 2$ and $\eta<0$. In the following we focus our attention on purely electric field configurations.

From the Maxwell equations (14) we obtain

$A_{t}^{\prime}(r)=\frac{Q}{(r(r+v))^{1 /(2 p-1)}}$,

where $Q$ is an integration constant related to $\tilde{Q}$ by $\tilde{Q}=$ $\eta 2^{p-1} p\left(Q^{2}\right)^{p} / Q$. So

$$
\begin{aligned}
A_{t}(r)= & \frac{(2 p-1) Q r^{(2-2 p) /(1-2 p)}(1 / v)^{1 /(-1+2 p)}}{2(p-1)}{ }_{2} F_{1} \\
& \times\left[\frac{1}{2 p-1}, \frac{2(p-1)}{2 p-1}, \frac{4 p-3}{2 p-1},-\frac{r}{v}\right]+C .
\end{aligned}
$$


The integration constant $C$ will be chosen in such a way the electric potential goes to zero asymptotically for $1 / 2<p<$ $3 / 2$, and is given by

$C=-Q(1 / v)^{\frac{3-2 p}{-1+2 p}} \frac{\Gamma\left(\frac{2-2 p}{1-2 p}\right) \Gamma\left(\frac{3-2 p}{-1+2 p}\right)}{\Gamma\left(\frac{1}{-1+2 p}\right)}$.

Thus, for $r \rightarrow \infty$ the electric potential behaves as

$A_{t}(r \rightarrow \infty) \approx Q \frac{1-2 p}{3-2 p} r^{\frac{3-2 p}{1-2 p}}+\cdots$,

where ... denotes terms that go to zero at large distances. Equation (22) shows that the field $A_{t}$ tends to zero at infinity for $1 / 2<p<3 / 2$ and diverges at infinity for $p<1 / 2$ and $p>3 / 2$. However, the above solution is not valid for $n=1 /(2 p-1)$ with $n>1$ being an integer. Fortunately, in that case we obtain analytical solutions that we present in the appendix. We can also determine the metric function $f(r)$ replacing (20) in (15). We find

$$
\begin{aligned}
f(r)= & -\frac{\Lambda}{3} r^{2}-\frac{1}{3} v\left(6 \alpha_{2}^{\prime}+\Lambda\right) r+k-\alpha_{2}^{\prime} v^{2} \\
& -2 \alpha_{2}^{\prime} r(r+v) \ln \left(\frac{r}{r+v}\right) \\
& +\frac{2 \alpha_{1} \eta 2^{p} p(2 p-1) v^{(1-4 p) /(1-2 p)}}{p-1} r(r+v) \\
& \times \int_{\infty}^{r} \frac{r^{2 p /(1-2 p)}}{(r+v)^{2}}{ }_{2} F_{1} \\
& \times\left[\frac{1}{2 p-1}, \frac{2(p-1)}{2 p-1}, \frac{4 p-3}{2 p-1},-\frac{r}{v}\right] \mathrm{d} r,
\end{aligned}
$$

where

$\alpha_{2}^{\prime}=\alpha_{2}-4 \alpha_{1} \eta 2^{p} p \frac{\Gamma\left(\frac{2-2 p}{1-2 p}\right) \Gamma\left(\frac{3-2 p}{-1+2 p}\right)}{\Gamma\left(\frac{1}{-1+2 p}\right)}$,

and the following redefinition of the integration constant $Q$ has been taken into account:

$\left(Q^{2}\right)^{p}=\frac{\alpha_{1}}{\left(v^{2}\right)^{2 p / 1-2 p}}$.

The scalar field potential can be written as

$$
\begin{aligned}
V(\phi)= & \frac{\Lambda}{3}(2+\cosh (\sqrt{2} \phi))+2 \alpha_{2}^{\prime}(-\sqrt{2} \phi[2 \\
& +\cosh (\sqrt{2} \phi)]+3 \sinh (\sqrt{2} \phi)) \\
& -2 \alpha_{1} \eta p 2^{p} \frac{2 p-1}{p-1}\left\{12\left(e^{\sqrt{2} \phi}-1\right)^{2(1-p) /(2 p-1)}\right. \\
& \times \sinh ^{2}(\phi / \sqrt{2}) \sinh (\sqrt{2} \phi) \\
& \times{ }_{2} F_{1}\left[\frac{1}{2 p-1}, \frac{2(p-1)}{2 p-1}, \frac{4 p-3}{2 p-1}, \frac{1}{1-e^{\sqrt{2} \phi}}\right]
\end{aligned}
$$

$$
\begin{aligned}
& +(2+\cosh (\sqrt{2} \phi)) F(\phi)\} \\
& +\alpha_{1} \eta 2^{p}\left(\frac{1}{4 \sinh ^{2}(\phi / \sqrt{2})}\right)^{2 p /(1-2 p)},
\end{aligned}
$$

where we have defined

$$
\begin{aligned}
F(\phi)= & v^{(1-4 p) /(1-2 p)} \int_{\infty}^{\frac{v}{-1+e^{\sqrt{2} \phi}}} \frac{r^{2 p /(1-2 p)}}{(r+v)^{2}}{ }_{2} F_{1} \\
& \times\left[\frac{1}{2 p-1}, \frac{2(p-1)}{2 p-1}, \frac{4 p-3}{2 p-1},-\frac{r}{v}\right] \mathrm{d} r \\
= & \int_{\infty}^{\frac{1}{-1+e^{\sqrt{2} \phi}}} \frac{r^{2 p /(1-2 p)}}{(r+1)^{2}}{ }_{2} F_{1} \\
& \times\left[\frac{1}{2 p-1}, \frac{2(p-1)}{2 p-1}, \frac{4 p-3}{2 p-1},-r\right] \mathrm{d} r .
\end{aligned}
$$

The integral above apparently depends on $v$; however, it can be shown numerically that the integral does not depend on $v$; so, in the last line of the above expression we have set $v=1$ for simplicity. Therefore, the potential $V(\phi)$ does not depend on the parameter $v$. We have used the reverse type procedure to obtain the potential, chosen in a way that the solution found from the remaining equations of motion with a metric ansatz and an electric field also solves the Klein-Gordon equation. Then we can investigate whether our system has a charged hairy black hole solution. In Fig. 1 we plot the behavior of the metric function $f(r)$ and the potential $V(\phi)$ in Fig. 2, for a choice of parameters $v=0.5, \Lambda=-1, \alpha_{1}=1$, $\alpha_{2}=1, p=2,3,5$, and $k= \pm 1,0$. Also, in Fig. 3 we plot the potential for other choices of the parameters. The metric function $f(r)$ changes sign for low values of $r$, signaling the presence of a horizon, while the potential tends to $\Lambda=V(0)$ $(V(0)<0)$ as can be seen in Figs. 2 and 3. We observe a different behavior of the potential, while it is bounded from below in Fig. 3, this does not occur in Fig. 2. It is worth to mention that potentials with similar behavior have been considered for instance in $[17,20,27,29]$. However, note that in Fig. 3 the shift of the minimum of the potential depends on the parameter $p$. Additionally, in Fig. 4 we plot the metric function $f(r)$ for other choices of the parameters, and we observe that for certain values of the parameters the metric can describe a black hole with two horizons, $r_{+}$and $r_{-}$. Moreover, the metric can describe an extremal black hole with degenerate horizons when $r_{+}=r_{-}$. Note that the full range of the $r$ coordinate covers all the values of $a(r)$ from the center $r=r_{c}$, where $a\left(r_{c}\right)=0$, to infinity [15]. So, for $v$ positive, the $r$ coordinate is in the range $0<r<\infty$, while for $v$ negative the range is $-v<r<\infty$. Finally, we have checked the behavior of the Kretschmann scalar $R_{\mu \nu \rho \sigma} R^{\mu \nu \rho \sigma}(r)$. Figure 5 shows that the Kretschmann scalar (with $v=0.5$ ) is singular at the center $r_{c}=0$, which is an essential property of these solutions, and the singularity is located in the dynamic region $\left(f\left(r_{c}\right)<0\right)$ for the numeri- 

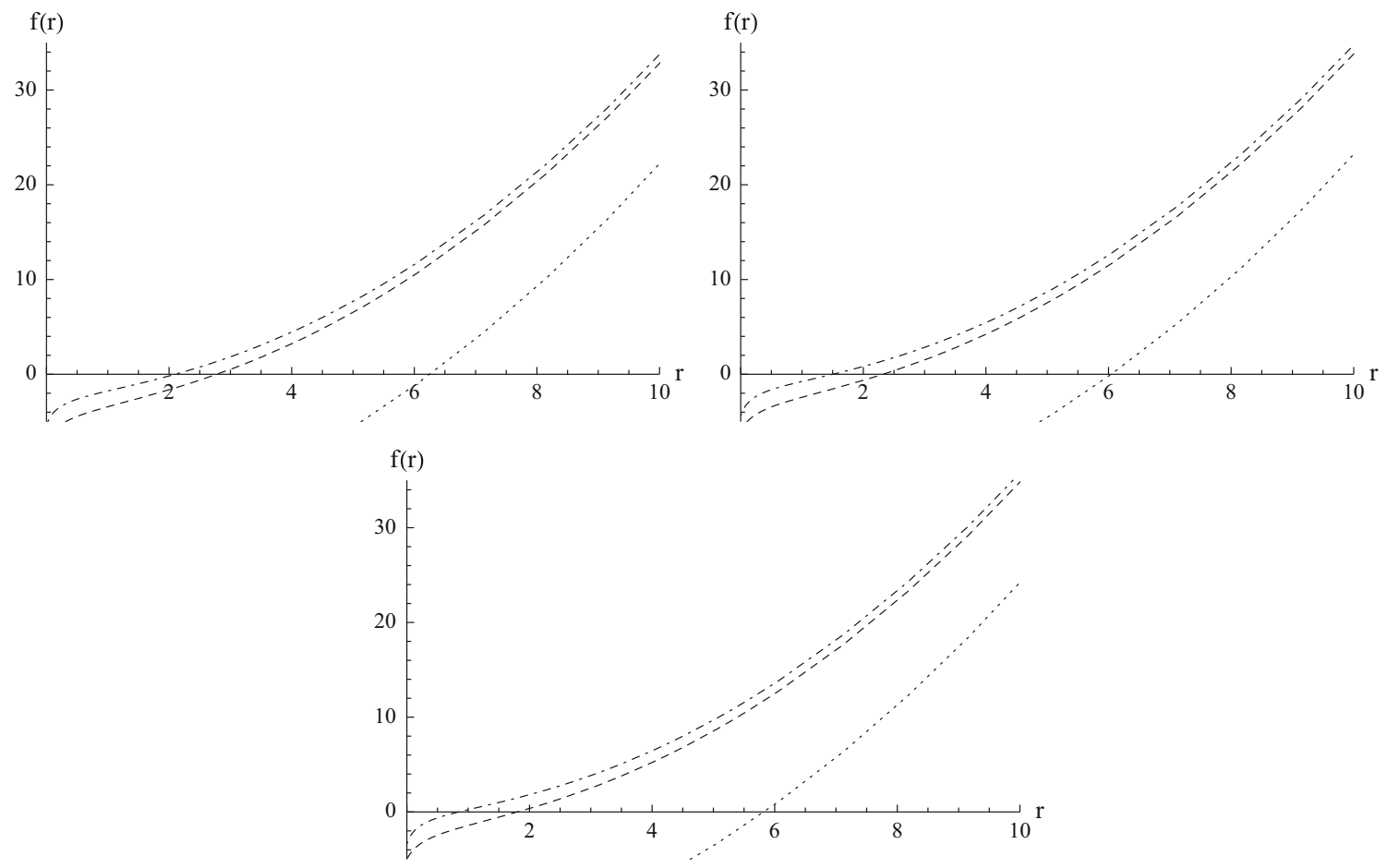

Fig. 1 The behavior of $f(r)$ for $v=0.5, \Lambda=-1, \alpha_{1}=1, \alpha_{2}=1, \eta=1 / 4, p=5$ (dotted line), $p=3$ (dashed line) and $p=2($ dot-dashed line). Left figure for $k=-1$, right figure for $k=0$, and bottom figure for $k=1$

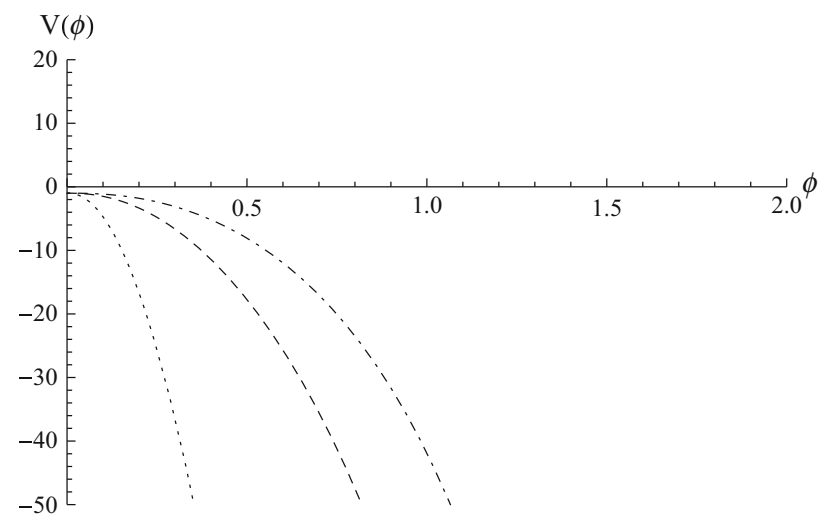

Fig. 2 The behavior of $V(\phi)$ for $\Lambda=-1, \alpha_{1}=1, \alpha_{2}=1, \eta=1 / 4$, $p=5$ (dotted line), $p=3$ (dashed line) and $p=2$ (dot-dashed line)

cal values considered (see Fig. 1). Furthermore, there is no curvature singularity outside the horizon; therefore, the metric (23) can describe a charged hairy black hole solution for certain values of the parameters. by

Equation (23) has an analytical solution for $p=3 / 4$ given

$$
\begin{aligned}
f(r)= & -\frac{\Lambda}{3} r^{2}-\frac{1}{3} v\left(6 \alpha_{2}+\Lambda\right) r+k-\alpha_{2} v^{2} \\
& -2 \alpha_{2} r(r+v) \ln \left(\frac{r}{r+v}\right) \\
& +3 \eta \alpha_{1} 2^{-1 / 4} \frac{\left(v(2 r+v)+2 r(r+v) \ln \frac{r}{r+v}\right)^{2}}{r(r+v)} .
\end{aligned}
$$

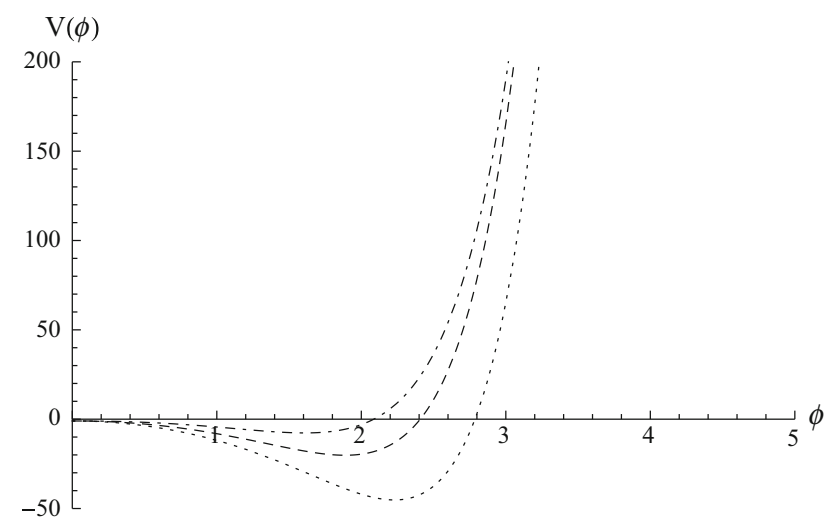

Fig. 3 The behavior of $V(\phi)$ for $\Lambda=-1, \alpha_{1}=0.1, \alpha_{2}=-5$, $\eta=1 / 4, p=3.5$ (dotted line), $p=3$ (dashed line) and $p=2$ (dot-dashed line)

The electric potential is

$A_{t}(r)=-\frac{Q}{v^{2}} \frac{2 r+v}{r(r+v)}-\frac{2 Q}{v^{3}} \ln \frac{r}{r+v}$

which goes to zero at infinity, and the self-interacting potential of the scalar field is

$$
\begin{aligned}
V(\phi)= & \frac{\Lambda}{3}(2+\cosh (\sqrt{2} \phi)) \\
& +2 \alpha_{2}(-\sqrt{2} \phi[2+\cosh (\sqrt{2} \phi)]+3 \sinh (\sqrt{2} \phi)) \\
& +64 \eta 2^{3 / 4} \alpha_{1} \sinh ^{6}(\phi / \sqrt{2})-3 \eta \alpha_{1} 2^{-1 / 4}
\end{aligned}
$$



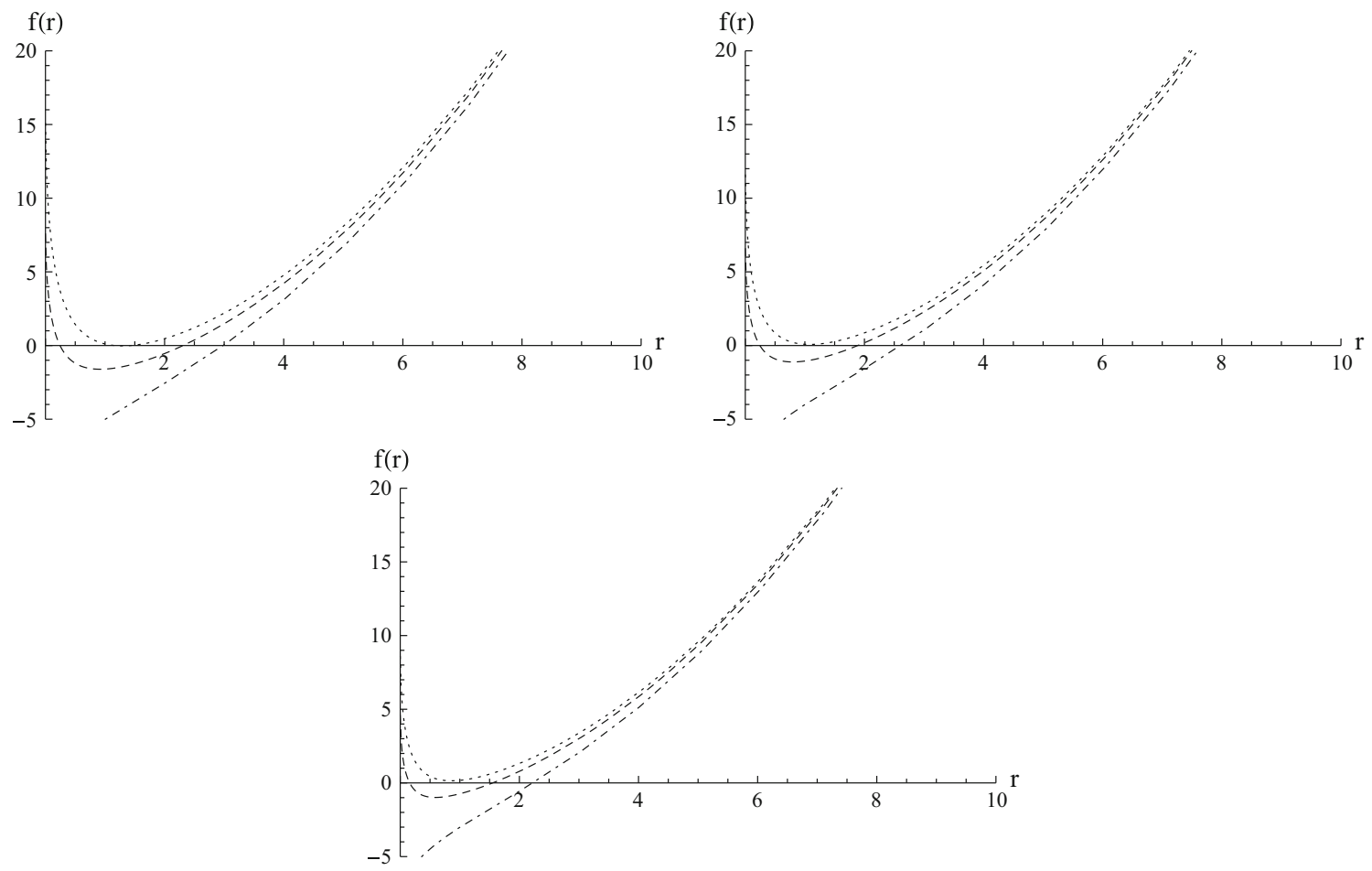

Fig. 4 The behavior of $f(r)$ for $v=1, \Lambda=-1, \alpha_{1}=1, \eta=1 / 4$, $p=2$. Left figure for $k=-1$ and $\alpha_{2}=-10$ (dot-dashed line), $\alpha_{2}=-25$ (dashed line), $\alpha_{2}=-32.5$ (dotted line). Right figure for $k=0$ and $\alpha_{2}=-10$ (dot-dashed line), $\alpha_{2}=-23$ (dashed line), $\alpha_{2}=-28$ (dotted line). Bottom figure for $k=1$ and $\alpha_{2}=-10$ (dotdashed line $), \alpha_{2}=-20$ (dashed line $), \alpha_{2}=-24$ (dotted line $)$
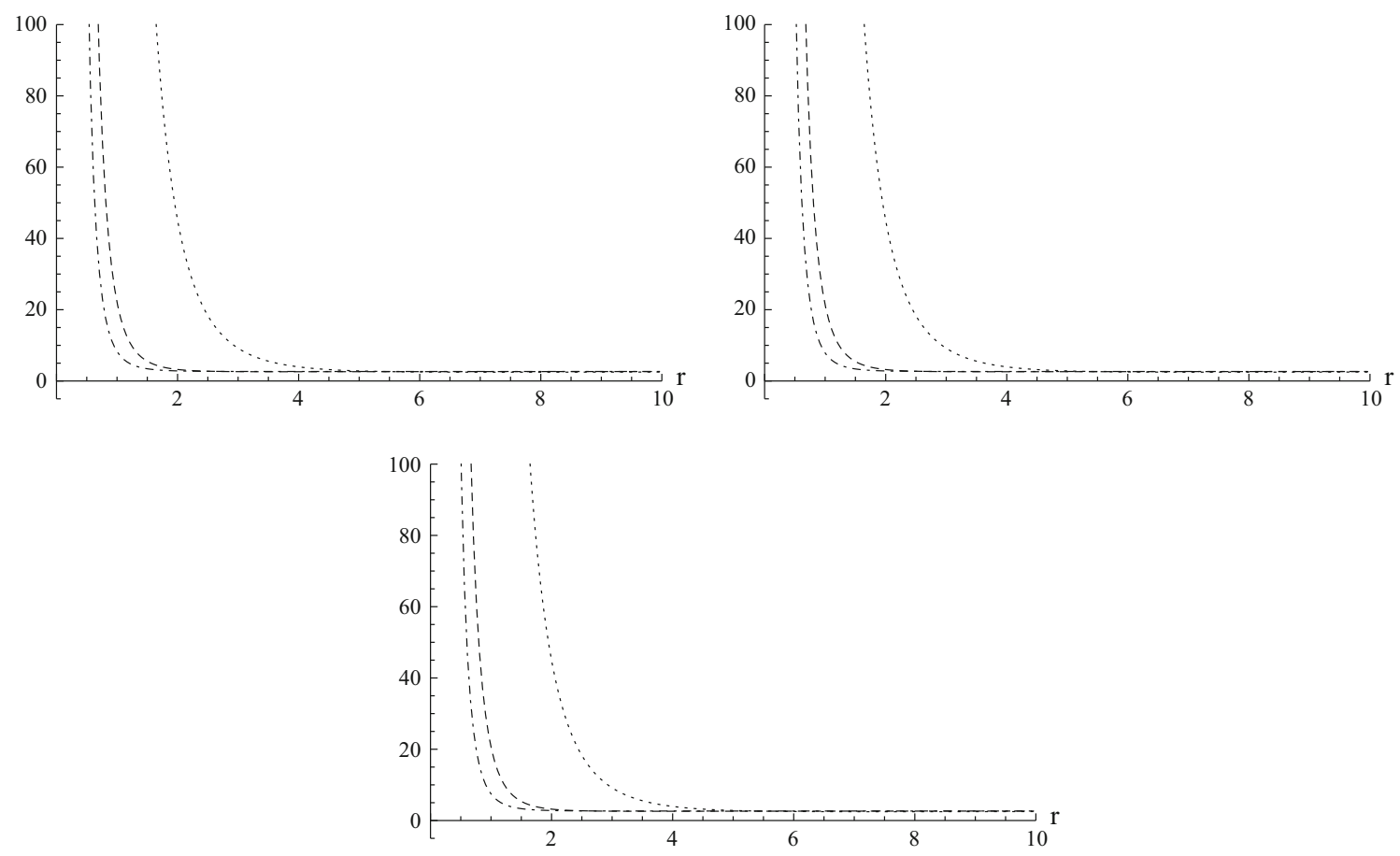

Fig. 5 The behavior of Kretschmann scalar $R_{\mu \nu \rho \sigma} R^{\mu \nu \rho \sigma}(r)$ as a function of $r$ for $v=0.5, \Lambda=-1, \alpha_{1}=1, \alpha_{2}=1, \eta=1 / 4, p=5($ dotted line), $p=3$ (dashed line) and $p=2$ (dot-dashed line). Left figure for $k=-1$, right figure for $k=0$, and bottom figure for $k=1$ 


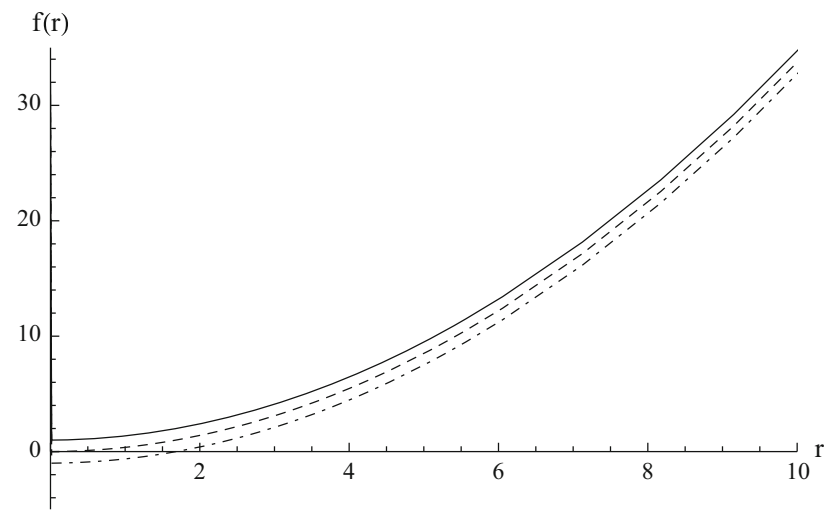

Fig. 6 The behavior of $f(r)$ for $v=1, \Lambda=-1, \eta=1 / 4, \alpha_{1}=1.5$, $\alpha_{2}=0.01, p=3 / 4, k=-1$ (dot-dashed line), $k=0$ (dashed line) and $k=1$ (continuous line)

$$
\begin{aligned}
& \times\left(-28+16 \phi^{2}+\left(31+8 \phi^{2}\right) \cosh (\sqrt{2} \phi)\right. \\
& -4 \cosh (2 \sqrt{2} \phi)+\cosh (3 \sqrt{2} \phi)-24 \sqrt{2} \phi \sinh (\sqrt{2} \phi)) .
\end{aligned}
$$

This solution is a special case of more general analytical solutions that we show in the appendix. In Fig. 6 we show the behavior of $f(r)$ for $p=3 / 4$ and different values of $k$.

\subsection{Born-Infeld type electrodynamics}

In this section we consider a Born-Infeld type Lagrangian given by

$\mathcal{L}\left(F^{2}\right)=4 b^{2}\left(1-\sqrt{1+\frac{F^{2}}{2 b^{2}}}\right)$,

where $b$ is the Born-Infeld coupling. In the limit $b \rightarrow \infty$ the Maxwell electrodynamics is recovered and in the limit $b \rightarrow 0$ this Lagrangian vanishes. By inserting (31) in (14), we obtain straightforwardly the electric field

$A_{t}^{\prime}(r)=\frac{\tilde{Q}}{\sqrt{r^{2}(r+v)^{2}+\frac{\tilde{Q}^{2}}{b^{2}}}}$.

Then, by performing the change of variable $u=r(r+v)+$ $v^{2} / 12$, the scalar potential reads

$A_{t}=\tilde{Q} \int \frac{\mathrm{d} u}{\sqrt{4 u^{3}-g_{2} u-g_{3}}}$,

where

$g_{2}=\frac{1}{12}\left(v^{4}-48 \frac{\tilde{Q}^{2}}{b^{2}}\right), \quad g_{3}=-\frac{v^{2}}{216}\left(144 \frac{\tilde{Q}^{2}}{b^{2}}+v^{4}\right)$.

Therefore, the solution for the scalar potential is

$A_{t}(r)=\tilde{Q}_{\wp}{ }^{-1}\left(r(r+v)+v^{2} / 12 ; g_{2}, g_{3}\right)$,

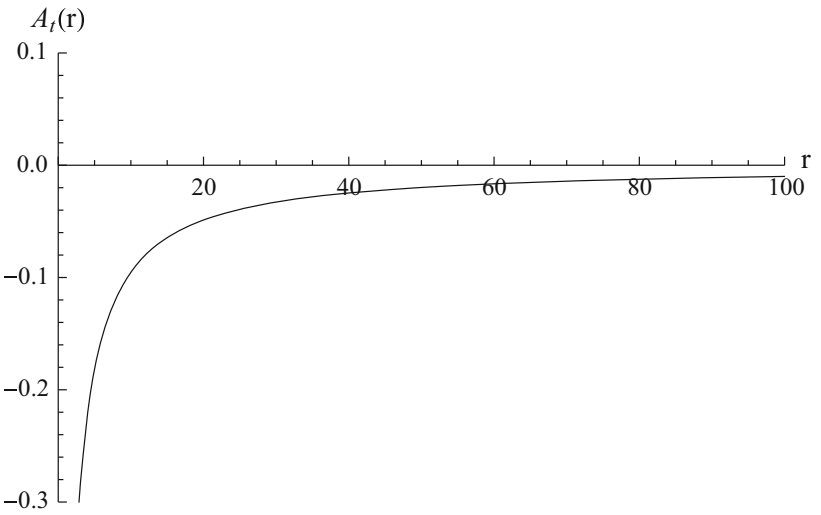

Fig. 7 The behavior of $A_{t}(r)$, for $b=1, v=1$, and $\tilde{Q}=1$

where $\wp$ denotes the $\wp$-Weierstrass elliptic function, with the Weierstrass invariants $g_{2}$ and $g_{3}$ given in (34). In Fig. 7 we plot $A_{t}(r)$ for $b=1, v=1$, and $\tilde{Q}=1$.

The metric function $f(r)$ reads

$$
\begin{aligned}
f(r)= & -\frac{\Lambda}{3} r^{2}-\frac{1}{3} v\left(6 \alpha_{2}+\Lambda\right) r+k-\alpha_{2} v^{2} \\
& -2 \alpha_{2} r(r+v) \ln \left(\frac{r}{r+v}\right)+8 v^{4} \alpha_{1}^{2} r(r+v) \\
& \times \int_{\infty}^{r} \frac{\wp^{-1}\left(r(r+v)+v^{2} / 12 ; g_{2}, g_{3}\right)}{r^{2}(r+v)^{2}} \mathrm{~d} r
\end{aligned}
$$

where we have taken into account the following redefinition:

$\tilde{Q}=\alpha_{1} v^{2}$,

and the potential can be written as

$$
\begin{aligned}
V(\phi)= & \frac{\Lambda}{3}(2+\cosh (\sqrt{2} \phi)) \\
& +2 \alpha_{2}(-\sqrt{2} \phi[2+\cosh (\sqrt{2} \phi)]+3 \sinh (\sqrt{2} \phi)) \\
& +4 b^{2}\left(1-\frac{b e^{\sqrt{2} \phi}}{\sqrt{\alpha_{1}^{2}\left(e^{\sqrt{2} \phi}-1\right)^{4}+b^{2} e^{2 \sqrt{2} \phi}}}\right) \\
& -64 \alpha_{1}^{2} \frac{\sinh ^{2}(\phi / \sqrt{2})}{\sqrt{\operatorname{csch}^{4}(\phi / \sqrt{2})+16 \alpha_{1}^{2} / b^{2}}} \\
& -8 \alpha_{1}^{2}\left(2+\cosh ^{2}(\sqrt{2} \phi)\right) F(\phi) \\
& -32 \alpha_{1}^{2} \sinh ^{2}(\phi / \sqrt{2}) \sinh (\sqrt{2} \phi) G(\phi),
\end{aligned}
$$

where we have defined

$$
\begin{aligned}
F(\phi) & =v^{4} \int_{\infty}^{\frac{v}{e^{\sqrt{2}} \phi-1}} \frac{\wp^{-1}\left(r(r+v)+v^{2} / 12 ; g_{2}, g_{3}\right)}{r^{2}(r+v)^{2}} \mathrm{~d} r \\
& =\int_{\infty}^{\frac{1}{e^{\sqrt{2}} \phi_{-1}}} \frac{\wp^{-1}\left(r(r+1)+1 / 12 ; g_{2}, g_{3}\right)}{r^{2}(r+1)^{2}} \mathrm{~d} r,
\end{aligned}
$$



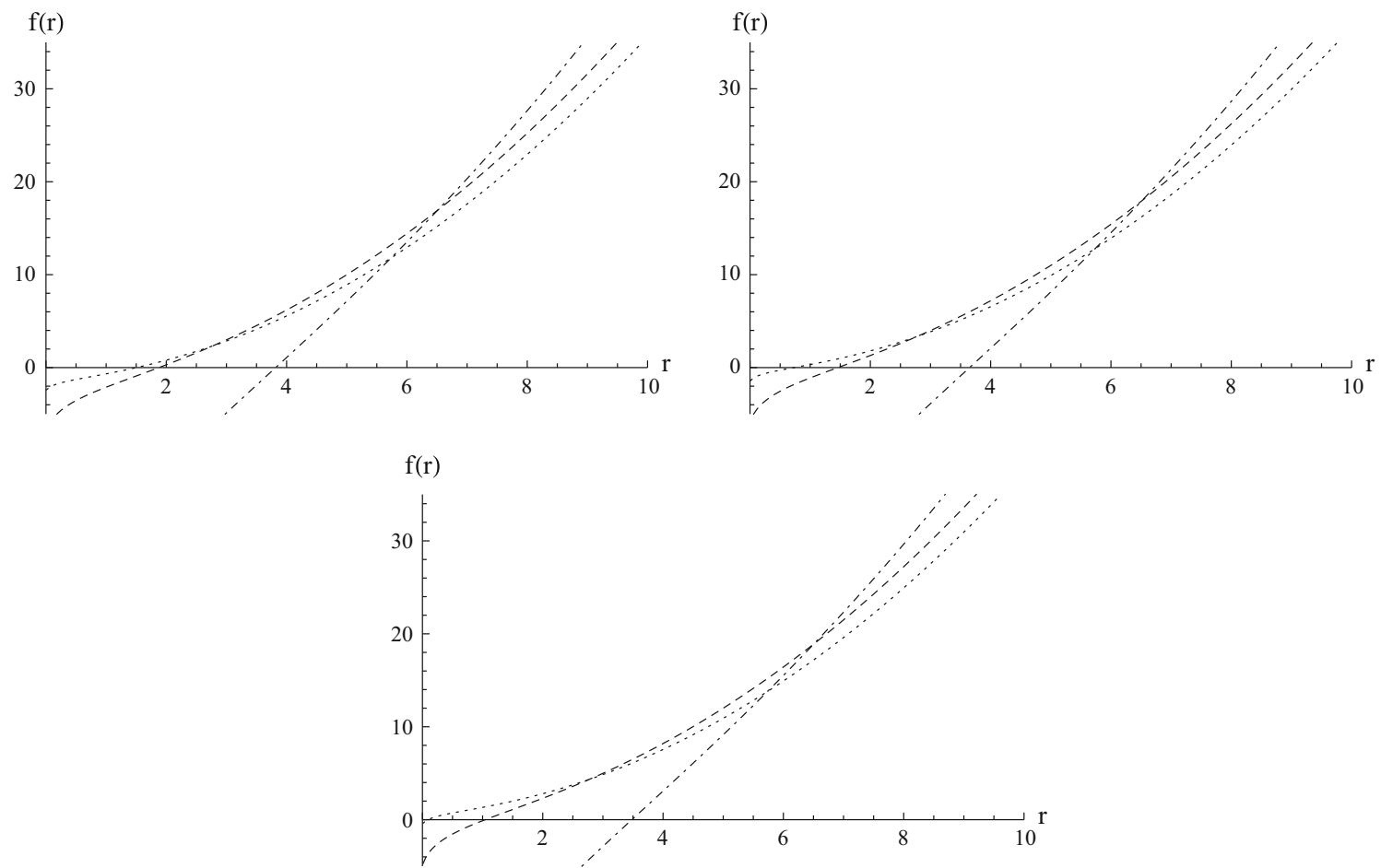

Fig. 8 The behavior of $f(r)$ for $\Lambda=-1, \alpha_{1}=0.01, \alpha_{2}=1.5, b=1, v=1$ (dotted line), $v=2$ (dashed line) and $v=5$ (dot-dashed line). Left figure for $k=-1$, right figure for $k=0$, and bottom figure for $k=1$

$$
\begin{aligned}
G(\phi) & =v \wp^{-1}\left(\frac{v^{2}}{4}\left(\operatorname{csch}^{2}(\phi / \sqrt{2})+1 / 3\right) ; g_{2}, g_{3}\right) \\
& =\wp^{-1}\left(\frac{1}{4}\left(\operatorname{csch}^{2}(\phi / \sqrt{2})+1 / 3\right) ; g_{2}, g_{3}\right) .
\end{aligned}
$$

Equation (39) apparently depends on the parameter $v$; however, it can be shown numerically that these expressions do not depend on $v$; for this reason, we have set $v=1$ in (39) for simplicity. Therefore, the potential $V(\phi)$ does not depend on the parameter $v$.

Then we can investigate whether our system has a charged hairy black hole solution. In Fig. 8 we plot the behavior of the metric function $f(r)$ and the potential $V(\phi)$ in Fig. 9, for a choice of parameters $\Lambda=-1, \alpha_{1}=0.01, \alpha_{2}=1.5, b=1$, and $v=1,2,5$. The metric function $f(r)$ changes sign for low values of $r$ signaling the presence of a horizon, while the potential tends to $\Lambda=V(0)(V(0)<0)$, as can be seen in Fig. 9. Additionally, in Fig. 10 we plot the metric function $f(r)$ for other choices of the parameters, and we observe that for certain values of the parameters the metric can describe a black hole with two horizons, $r_{+}$and $r_{-}$. Moreover, the metric can describe an extremal black hole with degenerate horizons when $r_{+}=r_{-}$. It is worth mentioning that in Fig. 10 we have set $v=-1$. As we mentioned in the previous section, for $v$ negative the full range of the $r$ coordinate is $-v<r<\infty$, and $r_{c}=-v$ corresponds to the center. It can be shown that the scalar field and the Kretschmann

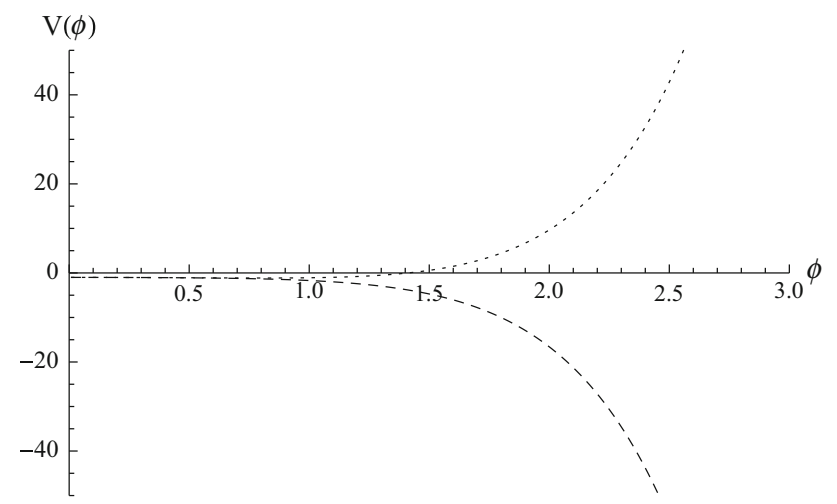

Fig. 9 The behavior of $V(\phi)$ for $\Lambda=-1, b=1, \alpha_{1}=0.01, \alpha_{2}=1.5$ (dashed line) and $\alpha_{2}=-1.5$ (dotted line)

scalar both diverge at the center $r_{c}=1$, which is an essential property of these solutions, and the singularity can be located either in a static region $\left(f\left(r_{c}\right)>0\right)$ as the dot-dashed and dashed lines illustrate in Fig. 10 or in the dynamic region $\left(f\left(r_{c}\right)<0\right)$ as the dotted lines show in Fig. 10. Additionally, in Fig. 11 we have plotted the behavior of the Kretschmann scalar $R_{\mu \nu \rho \sigma} R^{\mu \nu \rho \sigma}(r)$ for $v=0.5$, and it is shown that it is singular at the center $r_{c}=0$. The numerical results also show that there is no curvature singularity outside the horizon; therefore, the metric (36) can describe a charged hairy black hole solution for certain values of the parameters. 

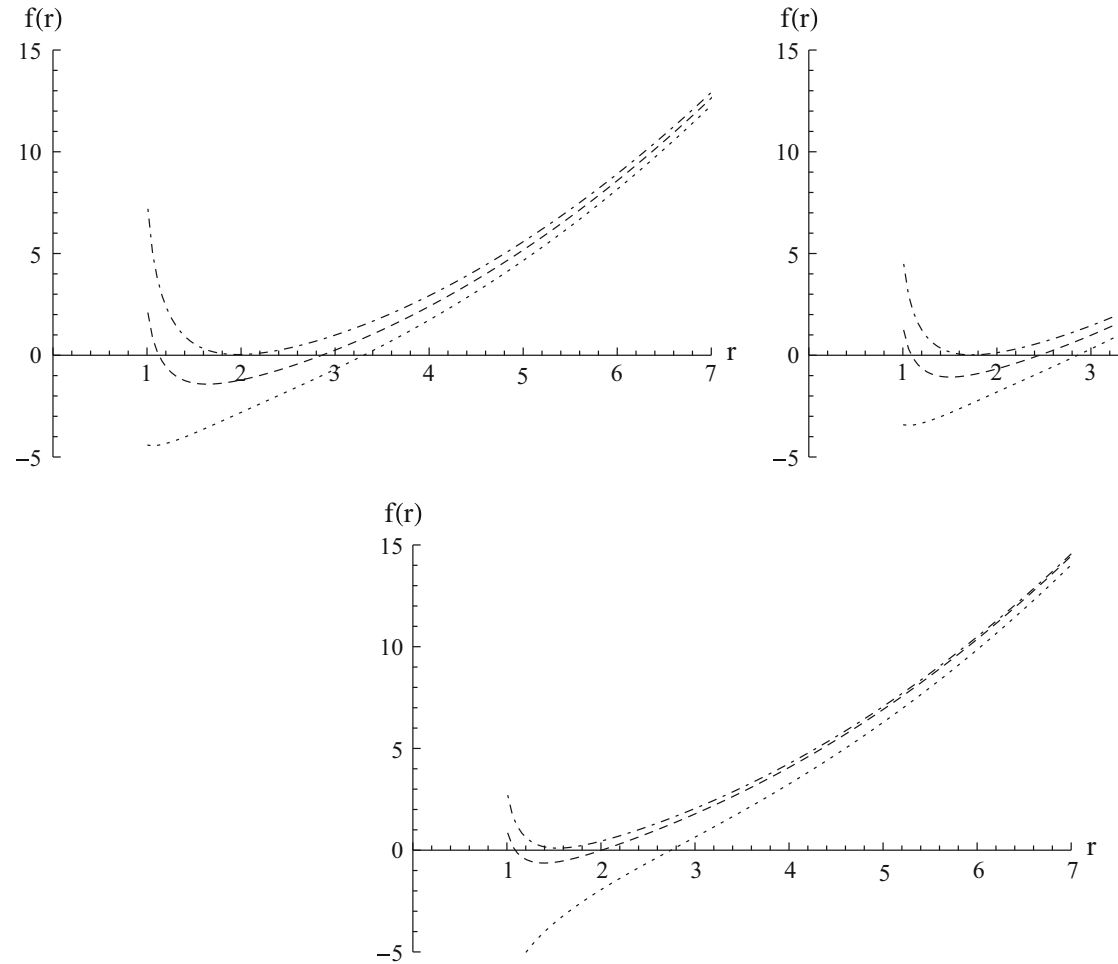

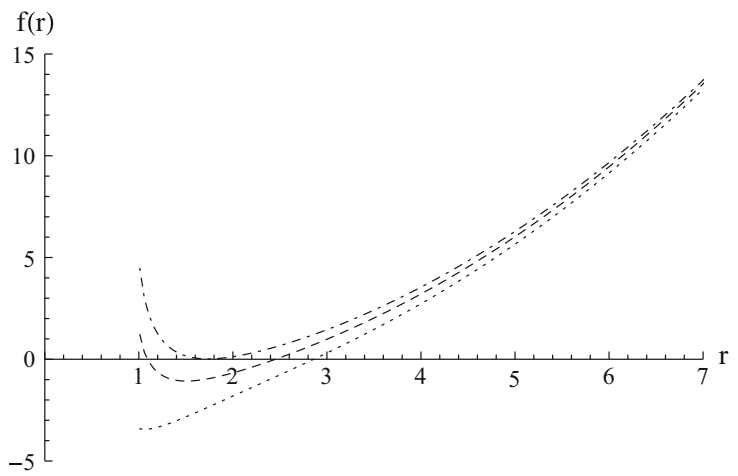

Fig. 10 The behavior of $f(r)$ for $\Lambda=-1, \alpha_{1}=1, v=-1, b=1$, (dotted line), $v=2$ (dashed line) and $v=5$ (dot-dashed line). Left figure for $k=-1$ and $\alpha_{2}=-15$ (dotted line), $\alpha_{2}=-8$ (dashed line), $\alpha_{2}=-2.5$ (dot-dashed line). Right figure for $k=0$ and $\alpha_{2}=-15$

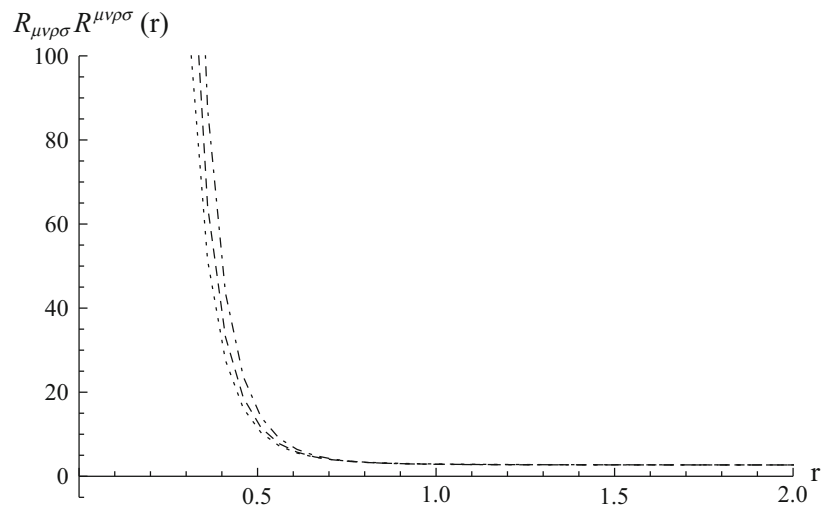

Fig. 11 The behavior of Kretschmann scalar $R_{\mu \nu \rho \sigma} R^{\mu \nu \rho \sigma}(r)$ as a function of $r$ for $v=0.5, \Lambda=-1, \alpha_{1}=1, \alpha_{2}=1, b=1, k=-1$ (dotted line), $k=0$ (dashed line $)$ and $k=1$ (dot-dashed line $)$

\section{Thermodynamics}

In this section we will study the thermodynamics of the hairy black hole solutions found. To compute the conserved charges we will apply the Euclidean formalism, we will work in the $\rho=\sqrt{r(r+v)}$ coordinate, in which the metric (5) can be written in the following form: (dotted line $), \alpha_{2}=-10$ (dashed line), $\alpha_{2}=-6.5$ (dot-dashed line). Bottom figure for $k=1$ and $\alpha_{2}=-20$ (dotted line), $\alpha_{2}=-11.5$ (dashed line), $\alpha_{2}=-9.5$ (dot-dashed line $)$

$\mathrm{d} s^{2}=-N(\rho)^{2} g(\rho)^{2} \mathrm{~d} t^{2}+\frac{1}{g(\rho)^{2}} \mathrm{~d} \rho^{2}+\rho^{2} \mathrm{~d} \Omega^{2}$,

where

$N(\rho)^{2}=\frac{\rho^{2}}{\left(\frac{v^{2}}{4}+\rho^{2}\right)}, \quad g(\rho)^{2}=\frac{f(\rho)}{\rho^{2}}\left(\frac{v^{2}}{4}+\rho^{2}\right)$,

and where $f(\rho)$ corresponds to the metric function $f(r)$ evaluated at $r=-\frac{v}{2}+\sqrt{\frac{\nu^{2}}{4}+\rho^{2}}$. In these coordinates the scalar field is given by

$\phi(\rho)=\frac{1}{\sqrt{2}} \ln \left[\frac{v+\sqrt{v^{2}+4 \rho^{2}}}{-v+\sqrt{v^{2}+4 \rho^{2}}}\right]$.

Now, we go to the Euclidean time $t \rightarrow i \tau$, and, for the Euclidean metric associated with (40), the Maxwell equation (13) yields

$\frac{A_{t}^{\prime}(\rho)}{N(\rho)} \frac{\mathrm{d} \mathcal{L}\left(F^{2}\right)}{\mathrm{d} F^{2}}=-\frac{\tilde{Q}}{\rho^{2}} ;$

here and in the following the prime ' denotes a derivative with respect to $\rho$. Thus, to apply the Euclidean formalism we consider the following action: 
$I_{E}=-\frac{\beta \Omega}{4 \pi} \int_{\rho+}^{\infty}\left(N(\rho) \mathcal{H}(\rho)+A_{t} \mathcal{P}^{\prime}\right) \mathrm{d} \rho+B$,

where

$$
\begin{aligned}
\mathcal{H}(\rho)= & \frac{\rho^{2}}{2 G}\left[\frac{g(\rho)^{2}-1+\rho\left(g(\rho)^{2}\right)^{\prime}}{\rho^{2}}\right. \\
& \left.+\left(\frac{1}{2} g(\rho)^{2} \phi^{\prime 2}+V(\phi)\right)-\left(\mathcal{L}\left(F^{2}\right)-\frac{4 A_{t}^{\prime}(\rho)}{N(\rho)} \frac{\tilde{Q}}{\rho^{2}}\right)\right]
\end{aligned}
$$

is the reduced Hamiltonian which satisfies the constraint $\mathcal{H}(\rho)=0$ and

$\mathcal{P}(\rho)=-\frac{4 A_{t}^{\prime}(\rho) \rho^{2}}{N(\rho)} \frac{\mathrm{d} \mathcal{L}\left(F^{2}\right)}{\mathrm{d} F^{2}}$,

which satisfies $\mathcal{P}^{\prime}(\rho)=0$. By using the Maxwell equation (43) we can show that $\mathcal{P}(\rho)=4 \tilde{Q}$ is a constant. Furthermore, $B$ is a surface term, $\beta=1 / T$ is the period of Euclidean time and finally $\Omega$ is the area of the spatial 2 section. We now compute the action when the field equations hold. The condition that the permitted geometries should not have conical singularities at the event horizon $\rho_{+}$imposes the requirement

$T=\frac{N\left(\rho_{+}\right)\left(g\left(\rho_{+}\right)^{2}\right)^{\prime}}{4 \pi}$.

So, by using the grand canonical ensemble we can fix the temperature and the electric potential $\Phi=-A_{t}\left(\rho_{+}\right)$. Then the variation of the surface term yields

$\delta B=\delta B_{G}+\delta B_{\phi}+\delta B_{F}$,

where

$$
\begin{aligned}
& \delta B_{G}=\left.\beta \Omega N(\rho) \rho \delta g(\rho)^{2}\right|_{\rho+} ^{\infty}, \\
& \delta B_{\phi}=\left.\beta \Omega N(\rho) \rho^{2} g(\rho)^{2} \phi^{\prime} \delta \phi\right|_{\rho+} ^{\infty}, \\
& \delta B_{F}=\left.\beta \Omega A_{t}(\rho) \delta \mathcal{P}\right|_{\rho+} ^{\infty} .
\end{aligned}
$$

For the variation of the fields at large distances we must be careful with the integral appearing in the metric function (15). It can be shown numerically that when $A_{t}$ tends to zero at infinity (for $1 / 2<p<3 / 2$ in power-law electrodynamics and in Born-Infeld type electrodynamics) the integral does not contribute to the conserved charges, and the variation of the fields at large distances yields

$$
\begin{aligned}
\delta B_{G \infty} & =\beta \Omega\left(-\frac{\Lambda v \rho}{6}-\alpha_{2} v^{2}+\mathcal{O}\left(\frac{1}{\rho}\right)\right) \delta v, \\
\delta B_{\phi \infty} & =\beta \Omega\left(\frac{\Lambda v \rho}{6}+\mathcal{O}\left(\frac{1}{\rho}\right)\right) \delta v \\
\delta B_{F \infty} & =\beta \Omega A_{t}(\infty) \delta \mathcal{P} .
\end{aligned}
$$

On the other hand, when the electric potential diverges at infinity (for $p>3 / 2$ in power-law electrodynamics) the variation of the integral in the metric function (15) diverges too. However, it can be verified numerically that it cancels out exactly with the contribution coming from $\delta B_{F \infty}=\beta \Omega A_{t}(\infty) \delta \mathcal{P}$. So, we can have well defined conserved charges in that case.

The variation of the fields at the horizon yields

$$
\begin{aligned}
\delta B_{G \rho_{+}} & =-\frac{4 \pi}{N(\rho) \beta} \delta \rho_{+}, \\
\delta B_{\phi \rho_{+}} & =0, \\
\delta B_{F \rho_{+}} & =\beta \Omega A_{t}\left(\rho_{+}\right) \delta \mathcal{P} .
\end{aligned}
$$

Therefore

$\delta B=-\frac{\beta \Omega}{3} \alpha_{2} \delta v^{3}+2 \pi \Omega \delta \rho_{+}^{2}+\beta \Omega \Phi \delta \mathcal{P}$.

Thus, as the Euclidean action is related to the free energy $F$ through $I_{E}=-\beta F$, we obtain

$I_{E}=S-\beta \mathcal{M}+\beta \Phi \mathcal{Q}$.

Then this relation makes it possible to identify the mass $(\mathcal{M})$, the entropy $(S)$ and the electric charge $(\mathcal{Q})$ as

$\mathcal{M}=\frac{\Omega}{3} \alpha_{2} v^{3}, \quad S=\frac{\Omega}{4 G} \rho_{+}^{2}, \mathcal{Q}=\Omega \mathcal{P}=4 \Omega \tilde{Q}$.

Thus, for power-law electrodynamics the electric charge is given by

$\mathcal{Q}_{(1)}=4 \Omega \eta 2^{p-1} p \alpha_{1}^{(2 p-1) / 2 p} v^{2}$,

and for the Born-Infeld type electrodynamics the electric charge is

$\mathcal{Q}_{(2)}=4 \Omega \alpha_{1} v^{2}$.

Having the temperature, mass, entropy, and electric charge it is possible to study phase transitions between the nonlinearly charged black holes with scalar hair and nonlinearly charged black holes without hair. For this analysis it is convenient to write the temperature of four-dimensional charged black holes with scalar hair (with $k=-1$ ) as

$$
\begin{aligned}
T= & \frac{1}{4 \pi}\left\{-\frac{1}{3}\left(2 r_{+} \Lambda+\left(12 \alpha_{2}+\Lambda\right) v\right)\right. \\
& -2 \alpha_{2}\left(2 r_{+}+v\right) \ln \left(\frac{r_{+}}{r_{+}+v}\right)+2^{2+p} \eta p\left(Q^{2}\right)^{p} / Q \\
& \times\left(\left(2 r_{+}+v\right) \int_{\infty}^{r_{+}} \frac{A_{t}(r)}{r^{2}(r+v)^{2}} \mathrm{~d} r\right. \\
& \left.\left.+r_{+}\left(r_{+}+v\right) \frac{A_{t}\left(r_{+}\right)}{r_{+}^{2}\left(r_{+}+v\right)^{2}}\right)\right\} .
\end{aligned}
$$

On the other hand, in the absence of a scalar field, the field equations have as a solution topological nonlinearly charged black holes, which correspond to the metric presented in 
Eq. (15) of Ref. [63] by setting the parameter $\alpha=0$ and $n=3$. The non-hairy black hole we consider here is also a generalization of the metric considered in [58], where a thermodynamic study of spherically symmetric black holes charged with power-law electrodynamics but with no cosmological constant was performed. The solution is

$$
\begin{aligned}
\mathrm{d} s^{2} & =-\tilde{f}(\rho) \mathrm{d} t^{2}+\frac{1}{\tilde{f}(\rho)} \mathrm{d} \rho^{2}+\rho^{2} \mathrm{~d} \Omega^{2}, \\
\tilde{f}(\rho) & =k-\frac{\Lambda}{3} \rho^{2}-\frac{m}{\rho}+\eta \frac{2^{p}(2 p-1)^{2}\left(q^{2}\right)^{p}}{(3-2 p) \rho^{\frac{2}{2 p-1}}}, \\
\tilde{A}_{t}(\rho) & =\frac{1-2 p}{3-2 p} q \rho^{\frac{3-2 p}{1-2 p}} .
\end{aligned}
$$

The temperature, entropy, mass, and electric charge are given, respectively, by

$$
\begin{aligned}
& T_{B H}=\frac{1}{4 \pi}\left(-\frac{1}{\rho_{+}}-\Lambda \rho_{+}+\eta 2^{p}(1-2 p)\left(q^{2}\right)^{p} \rho_{+}^{\frac{1+2 p}{1-2 p}}\right) \\
& S_{B H}=2 \pi \Omega \rho_{+}^{2} \\
& M_{B H}=-\Omega \rho_{+}\left(1+\frac{\rho_{+}^{2} \Lambda}{3}-\eta \frac{2^{p}(2 p-1)^{2} \rho_{+}^{\frac{2}{1-2 p}}\left(q^{2}\right)^{p}}{3-2 p}\right) \\
& Q_{B H}=2 \eta \frac{\Omega p 2^{p}\left(q^{2}\right)^{p}}{q}
\end{aligned}
$$

where

$q=\frac{(2 p-3) \Phi}{(1-2 p) \rho_{+}^{\frac{3-2 p}{1-2 p}}}$.

So, the horizon radius $\rho_{+}$can be written as a function of the temperature and of the electric potential. Now, in order to find phase transitions, we must consider both black holes in the same grand canonical ensemble, i.e., at the same $T$ and $\Phi$. Making $T$ and $\Phi$ equal for both black holes and by considering the free energy $F$,

$F=F(T, \Phi)=\mathcal{M}-T S-\Phi \mathcal{Q}$,

we plot the difference of the free energies for the nonlinearly charged black hole $\left(F_{1}\right)$ and the nonlinearly charged black hole with scalar hair $\left(F_{0}\right), \Delta F=F_{1}-F_{0}$ as a function of the temperature in Figs. 12 and 13. In Fig. 12 we show that there is a second-order phase transition at the fixed critical temperature $T_{c} \approx 0.16$ for low values of $p$, and the topological nonlinearly charged hairy black hole dominates for temperatures lower than critical, whereas for temperatures higher than critical the topological nonlinearly charged black hole is thermodynamically preferred. In Fig. 13 we show that there are no phase transitions for high values of $p$ and the charged black hole without scalar hair is thermodynamically preferred. It is worth mentioning that since $v$ and $\tilde{Q}$ are related, the temperature and the electric potential are not independent; thus, we can express the free energy as a

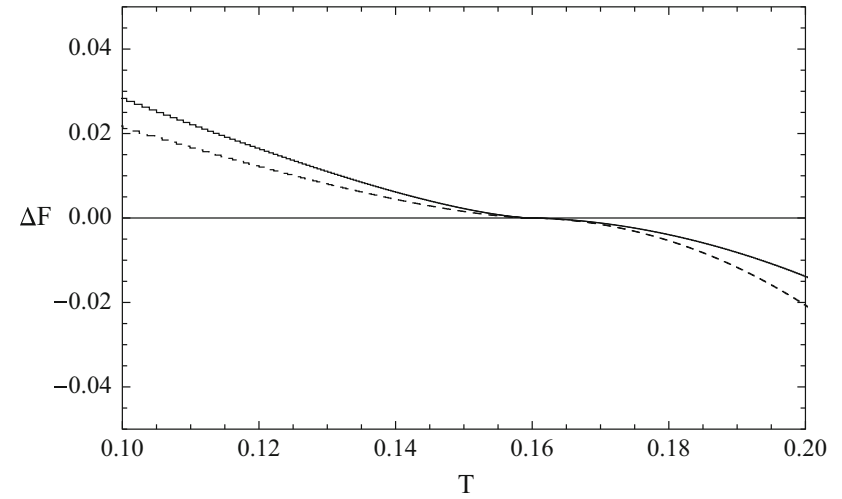

Fig. 12 The behavior of $\Delta F=F_{1}-F_{0}$ as a function of the temperature $T$ with $k=-1, \Omega=1, \Lambda=-3, \alpha_{1}=0.05, \alpha_{2}=-1, \eta=0.5$ with $p=0.9$ (continuous line $)$ and $p=1.2($ dashed line $)$

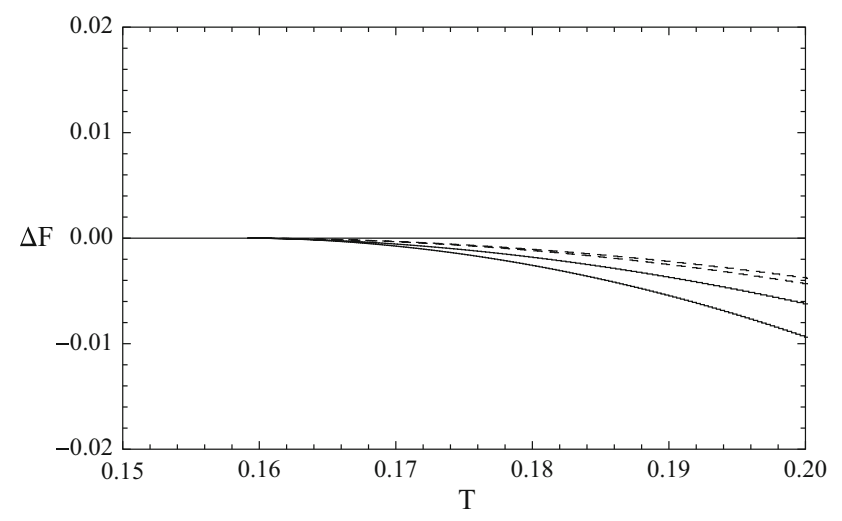

Fig. 13 The behavior of $\Delta F=F_{1}-F_{0}$ as a function of the temperature $T$ with $k=-1, \Omega=1, \Lambda=-3, \alpha_{1}=0.05, \alpha_{2}=-1, \eta=0.5$ with $p=2$ (continuous line) and $p=3$ (dashed line)

function of $T$ or $\Phi$ only. This is similar to what happens in [85] for a charged hairy black hole in linear electrodynamics.

\section{Conclusions}

We have considered a gravitating system consisting of a scalar field minimally coupled to gravity with a selfinteracting potential and a $\mathrm{U}(1)$ nonlinear electromagnetic field. We solved the coupled field equations with a profile of the scalar field which falls sufficiently fast outside the black hole horizon. For a range of values of the scalar field parameter, which characterizes its behavior, we found numerically charged hairy black hole solutions in power-law and Born-Infeld type electrodynamics, with the scalar field regular everywhere outside and on the event horizon. Also, in the case of power-law electrodynamics, we found analytical hairy black hole solutions for some special values of the exponent $p$ in the range $1 / 2<p<1$. Then we studied the thermodynamics for both electrodynamics and phase transitions 
of our black hole solutions in power-law electrodynamics, and we showed that there is a second-order phase transition for low values of $p$. Moreover, at a low temperature, the topological nonlinearly charged hairy black hole is thermodynamically preferred, whereas the topological charged black hole without scalar hair is thermodynamically preferred at a high temperature for power-law electrodynamics. Interestingly enough, these phase transitions occur at a fixed critical temperature and do not depend on the exponent $p$ of the nonlinearity electrodynamics. On the other hand, we showed that there is no phase transition for high values of $p$. This picture is consistent with the findings of the application of the AdS/CFT correspondence to condensed matter systems. In these systems there is a critical temperature below which the system undergoes a phase transition to a hairy black hole configuration at a low temperature. This corresponds in the boundary field theory to the formation of a condensation of the scalar field.

Acknowledgements We would like to thank the anonymous referee for valuable comments which help us to improve the quality of our paper. This work was partially funded by Comisión Nacional de Ciencias y Tecnología through FONDECYT Grants 11140674 (PAG), by Dirección de Investigación y Desarrollo de la Universidad de La Serena (Y.V.) and partially supported by grants from Comisión Nacional de Investigación Científica y Tecnológica CONICYT, Doctorado Nacional 2016 (21160784) (J.B.O.). P.A.G. and J.B.O. acknowledge the hospitality of the Universidad de La Serena, where part of this work was carried out. P.A.G. acknowledges the hospitality of the National Technical University of Athens.

Open Access This article is distributed under the terms of the Creative Commons Attribution 4.0 International License (http://creativecomm ons.org/licenses/by/4.0/), which permits unrestricted use, distribution, and reproduction in any medium, provided you give appropriate credit to the original author(s) and the source, provide a link to the Creative Commons license, and indicate if changes were made. Funded by SCOAP ${ }^{3}$.

\section{Appendix A: Analytical solutions}

In this appendix we present some analytical hairy black hole solutions with power-law electrodynamics for some particular values of the exponent $p$. For $p=\frac{1}{2}+\frac{1}{2 n}$, with $n>1$ being an integer number, so $\frac{1}{2}<p<1$, the electric field is given by

$$
A_{t}(r)=Q I 1(r ; n),
$$

where we have defined

$$
\begin{aligned}
I 1(r ; n) \equiv & \int \frac{\mathrm{d} r}{(r(r+v))^{n}}=\frac{\frac{v}{2}+r}{-\frac{v^{2}}{2}(n-1)(r(r+v))^{n-1}} \\
& +\frac{\frac{v}{2}+r}{(n-1)} \sum_{j=1}^{n-2}\left(\prod_{i=1}^{j} \frac{2 n-2 i-1}{n-i-1}\right)
\end{aligned}
$$

$$
\begin{aligned}
& \times \frac{1}{\left(-\frac{v^{2}}{2}\right)^{j+1}(r(r+v))^{n-j-1}} \\
& +\frac{1}{v\left(-\frac{v^{2}}{2}\right)^{n-1}} \prod_{i=1}^{n-1} \frac{2 n-2 i-1}{n-i} \ln \left(\frac{r}{r+v}\right) .
\end{aligned}
$$

So, using this expression, we find that the metric function is given by

$$
\begin{aligned}
& f(r)=-\frac{\Lambda}{3} r^{2}-\frac{1}{3} v\left(6 \alpha_{2}+\Lambda\right) r+k-\alpha_{2} v^{2} \\
& -2 \alpha_{2} r(r+v) \ln \left(\frac{r}{r+v}\right) \\
& +2^{\frac{3}{2}+\frac{1}{2 n}} \eta \frac{(n+1)}{n} Q^{\frac{(n+1)}{n}}\left\{-r(r+v) \frac{1}{\frac{v^{2}}{2}(n-1)}\right. \\
& \times\left(\frac{v}{2} I 1(r ; n+1)+I 2(r ; n+1)\right) \\
& +r(r+v) \sum_{j=1}^{n-2}\left(\prod_{i=1}^{j} \frac{2 n-2 i-1}{n-i-1}\right) \frac{1}{(n-1)\left(-\frac{v^{2}}{2}\right)^{j+1}} \\
& \times\left(\frac{v}{2} I 1(r ; n-j+1)+I 2(r ; n-j+1)\right) \\
& -\frac{1}{\left(-\frac{v^{2}}{2}\right)^{n-1} v^{4}}\left(\prod_{i=1}^{n-1} \frac{2 n-2 i-1}{n-i}\right) \\
& \left.\times\left(v+(r+v) \ln \left(\frac{r}{r+v}\right)\right)\left(v+r \ln \left(\frac{r}{r+v}\right)\right)\right\} \text {, }
\end{aligned}
$$

where we have defined

$$
\begin{aligned}
I 2(r ; n) \equiv & \int \frac{r \mathrm{~d} r}{(r(r+v))^{n}}=\frac{r}{v(n-1)(r(r+v))^{n-1}} \\
& +\frac{2 n-3}{v(n-1)} I 1(r ; n-1) .
\end{aligned}
$$

On the other hand, for $p=\frac{1}{2}+\frac{1}{2 m-1}$, with $m>1$ being an integer number, so $\frac{1}{2}<p<1$, the electric field is given by

$A_{t}(r)=Q I 3(r ; m)$,

where

$$
\begin{aligned}
I 3(r ; m) \equiv & \int \frac{\mathrm{d} r}{(r(r+v))^{\frac{2 m-1}{2}}}=\frac{\frac{v}{2}+r}{-\frac{v^{2}}{2} \frac{2 m-3}{2}(r(r+v))^{\frac{2 m-3}{2}}} \\
& +\frac{\frac{v}{2}+r}{\frac{2 m-3}{2}} \sum_{j=1}^{m-1}\left(\prod_{i=1}^{j} \frac{2 m-2 i-2}{\frac{2 m-1}{2}-i-1}\right) \\
& \times \frac{1}{\left(-\frac{v^{2}}{2}\right)^{j+1}(r(r+v))^{\frac{2 m-2 j-3}{2}}},
\end{aligned}
$$


and the metric function is

$$
\begin{aligned}
f(r)= & -\frac{\Lambda}{3} r^{2}-\frac{1}{3} v\left(6 \alpha_{2}+\Lambda\right) r+k-\alpha_{2} v^{2} \\
& -2 \alpha_{2} r(r+v) \ln \left(\frac{r}{r+v}\right) \\
& +2^{\frac{3}{2}+\frac{1}{2 m-1}} \eta \frac{2 m+1}{2 m-1} Q^{\frac{2 m+1}{2 m-1}}\left\{-r(r+v) \frac{1}{\frac{v^{2}}{2}(2 m-3)}\right. \\
& \times\left(\frac{v}{2} I 3(r ; m+1)+I 4(r ; m+1)\right) \\
& +r(r+v) \sum_{j=1}^{m-1}\left(\prod_{i=1}^{j} \frac{2 m-2 i-2}{\frac{2 m-1}{2}-i-1}\right) \\
& \times \frac{1}{\left(\frac{2 m-3}{2}\right)\left(-\frac{v^{2}}{2}\right)^{j+1}} \\
& \left.\times\left(\frac{v}{2} I 3(r ; m-j+1)+I 4(r ; m-j+1)\right)\right\},
\end{aligned}
$$

where

$$
\begin{aligned}
I 4(r ; m) \equiv & \int \frac{r \mathrm{~d} r}{(r(r+v))^{\frac{2 m-1}{2}}}=\frac{r}{\frac{2 m-3}{2} v(r(r+v))^{\frac{2 m-3}{2}}} \\
& +\frac{2(m-2)}{\frac{2 m-3}{2} v} I 3(r ; m-1) .
\end{aligned}
$$

\section{References}

1. N. Bocharova, K. Bronnikov, V. Melnikov, Vestn. Mosk. Univ. Fiz. Astron. 6, 706 (1970)

2. J.D. Bekenstein, Ann. Phys. 82, 535 (1974)

3. J.D. Bekenstein, Black holes with scalar charge. Ann. Phys. 91, 75 (1975)

4. K.A. Bronnikov, Y.N. Kireyev, Instability of black holes with scalar charge. Phys. Lett. A 67, 95 (1978)

5. O. Bechmann, O. Lechtenfeld, Class. Quantum Gravity 12, 1473 (1995). arXiv:gr-qc/9502011

6. H. Dennhardt, O. Lechtenfeld, Int. J. Mod. Phys. A 13, 741 (1998). arXiv:gr-qc/9612062

7. K.G. Zloshchastiev, On co-existence of black holes and scalar field. Phys. Rev. Lett. 94, 121101 (2005). arXiv:hep-th/0408163

8. T. Torii, K. Maeda, M. Narita, No-scalar hair conjecture in asymptotic de Sitter spacetime. Phys. Rev. D 59, 064027 (1999). arXiv:gr-qc/9809036

9. C. Martinez, R. Troncoso, J. Zanelli, De Sitter black hole with a conformally coupled scalar field in four-dimensions. Phys. Rev. D 67, 024008 (2003). arXiv:hep-th/0205319

10. T.J.T. Harper, P.A. Thomas, E. Winstanley, P.M. Young, Instability of a four-dimensional de Sitter black hole with a conformally coupled scalar field. Phys. Rev. D 70, 064023 (2004). arXiv:gr-qc/0312104

11. G. Dotti, R.J. Gleiser, C. Martinez, Static black hole solutions with a self interacting conformally coupled scalar field. Phys. Rev. D 77, 104035 (2008). arXiv:0710.1735 [hep-th]

12. T. Torii, K. Maeda, M. Narita, Scalar hair on the black hole in asymptotically anti-de Sitter spacetime. Phys. Rev. D 64, 044007 (2001)
13. E. Winstanley, On the existence of conformally coupled scalar field hair for black holes in (anti-)de Sitter space. Found. Phys. 33, 111 (2003). arXiv:gr-qc/0205092

14. C. Martinez, R. Troncoso, J. Zanelli, Exact black hole solution with a minimally coupled scalar field. Phys. Rev. D 70, 084035 (2004). arXiv:hep-th/0406111

15. K.A. Bronnikov, Phys. Rev. D 64, 064013 (2001). doi:10.1103/ PhysRevD.64.064013. arXiv:gr-qc/0104092

16. A. Anabalon, Exact black holes and universality in the backreaction of non-linear sigma models with a potential in (A)dS4. JHEP 1206, 127 (2012). arXiv:1204.2720 [hep-th]

17. A. Anabalon, J. Oliva, Exact hairy black holes and their modification to the universal law of gravitation. Phys. Rev. D 86, 107501 (2012). arXiv:1205.6012 [gr-qc]

18. A. Anabalon, A. Cisterna, Asymptotically (anti) de Sitter black holes and wormholes with a self interacting scalar field in four dimensions. Phys. Rev. D 85, 084035 (2012). arXiv:1201.2008 [hep-th]

19. Y. Bardoux, M.M. Caldarelli, C. Charmousis, Conformally coupled scalar black holes admit a flat horizon due to axionic charge. JHEP 1209, 008 (2012). arXiv:1205.4025 [hep-th]

20. P.A. González, E. Papantonopoulos, J. Saavedra, Y. Vásquez, JHEP 1312, 021 (2013). arXiv:1309.2161 [gr-qc]

21. T. Kolyvaris, G. Koutsoumbas, E. Papantonopoulos, G. Siopsis, Scalar hair from a derivative coupling of a scalar field to the Einstein tensor. Class. Quantum Gravity 29, 205011 (2012). arXiv:1111.0263 [gr-qc]

22. T. Kolyvaris, G. Koutsoumbas, E. Papantonopoulos, G. Siopsis, Phase transition to a hairy black hole in asymptotically flat spacetime. JHEP 1311, 133 (2013). arXiv:1308.5280 [hep-th]

23. A. Anabalon, A. Cisterna, J. Oliva, Phys. Rev. D 89, 084050 (2014). arXiv:1312.3597 [gr-qc]

24. A. Cisterna, C. Erices, Phys. Rev. D 89, 084038 (2014). arXiv:1401.4479 [gr-qc]

25. C. Martinez, J.P. Staforelli, R. Troncoso, Topological black holes dressed with a conformally coupled scalar field and electric charge. Phys. Rev. D 74, 044028 (2006). arXiv:hep-th/0512022

26. C. Martinez, R. Troncoso, Electrically charged black hole with scalar hair. Phys. Rev. D 74, 064007 (2006). arXiv:hep-th/0606130

27. P.A. Gonzalez, E. Papantonopoulos, J. Saavedra, Y. Vasquez, JHEP 1411, 011 (2014). arXiv:1408.7009 [gr-qc]

28. W. Xu, D.C. Zou, arXiv:1408.1998 [hep-th]

29. Z.Y. Fan, H. Lu, JHEP 1509, 060 (2015). arXiv:1507.04369 [hepth]

30. O.J.C. Dias, G.T. Horowitz, J.E. Santos, Black holes with only one Killing field. JHEP 1107, 115 (2011). arXiv:1105.4167 [hep-th]

31. S. Stotyn, M. Park, P. McGrath, R.B. Mann, Black holes and boson stars with one Killing field in arbitrary odd dimensions. Phys. Rev. D 85, 044036 (2012). arXiv:1110.2223 [hep-th]

32. O.J.C. Dias, P. Figueras, S. Minwalla, P. Mitra, R. Monteiro, J.E. Santos, Hairy black holes and solitons in global $A d S_{5}$. JHEP 1208, 117 (2012). arXiv: 1112.4447 [hep-th]

33. B. Kleihaus, J. Kunz, E. Radu, B. Subagyo, Axially symmetric static scalar solitons and black holes with scalar hair. Phys. Lett. B 725, 489 (2013). arXiv:1306.4616 [gr-qc]

34. C.A.R. Herdeiro, E. Radu, Kerr black holes with scalar hair. Phys. Rev. Lett. 112, 221101 (2014). arXiv:1403.2757 [gr-qc]

35. C.A.R. Herdeiro, E. Radu. A new spin on black hole hair. Int. J. Mod. Phys. D 23(12), 1442014 (2014). doi:10.1142/ S0218271814420140. arXiv:1405.3696 [gr-qc]

36. X. Zhang, H. Lu, Phys. Lett. B 736, 455 (2014). arXiv:1403.6874 [hep-th]

37. H. Lü, X. Zhang, JHEP 1407, 099 (2014). arXiv:1404.7603 [hepth]

38. M.S. Volkov, arXiv:1601.08230 [gr-qc]

39. M. Born, L. Infeld, Proc. R. Soc. Lond. A 144, 425 (1934) 
40. Y. Kats, L. Motl, M. Padi, JHEP 0712, 068 (2007). arXiv:hep-th/0606100

41. D. Anninos, G. Pastras, JHEP 0907, 030 (2009). arXiv:0807.3478 [hep-th]

42. R.G. Cai, Z.Y. Nie, Y.W. Sun, Phys. Rev. D 78, 126007 (2008). arXiv:0811.1665 [hep-th]

43. E. Ayon-Beato, A. Garcia, Phys. Rev. Lett. 80, 5056 (1998). arXiv:gr-qc/9911046

44. E. Ayon-Beato, A. Garcia, Phys. Lett. B 464, 25 (1999). arXiv:hep-th/9911174

45. M. Cataldo, A. Garcia, Phys. Rev. D 61, 084003 (2000). arXiv:hep-th/0004177

46. K.A. Bronnikov, Phys. Rev. D 63, 044005 (2001). arXiv:gr-qc/0006014

47. A. Burinskii, S.R. Hildebrandt, Phys. Rev. D 65, 104017 (2002). arXiv:hep-th/0202066

48. J. Matyjasek, Phys. Rev. D 70, $047504 \quad$ (2004). arXiv:gr-qc/0403109

49. R.G. Cai, D.W. Pang, A. Wang, Phys. Rev. D 70, 124034 (2004). arXiv:hep-th/0410158

50. T.K. Dey, Phys. Lett. B 595, 484 (2004). arXiv:hep-th/0406169

51. M. Aiello, R. Ferraro, G. Giribet, Phys. Rev. D 70, 104014 (2004). arXiv:gr-qc/0408078

52. S.H. Hendi, Phys. Lett. B 690, 220 (2010). arXiv:0907.2520 [gr-qc]

53. S.H. Hendi, Eur. Phys. J. C 69, 281 (2010). arXiv:1008.0168 [hepth]

54. S.H. Hendi, Ann. Phys. 333, 282 (2013). arXiv:1405.5359 [gr-qc]

55. S.H. Hendi, B.E. Panah, S. Panahiyan, JHEP 1511, 157 (2015). arXiv:1508.01311 [hep-th]

56. S.H. Hendi, S. Panahiyan, B.E. Panah, M. Momennia, Eur. Phys. J. C 76(3), 150 (2016)

57. O. Miskovic, R. Olea, Phys. Rev. D 77, 124048 (2008). arXiv:0802.2081 [hep-th]

58. H.A. Gonzalez, M. Hassaine, C. Martinez, Phys. Rev. D 80, 104008 (2009). arXiv:0909.1365 [hep-th]

59. S.H. Hendi, B.E. Panah, Phys. Lett. B 684, 77 (2010). arXiv: 1008.0102 [hep-th]

60. A. Sheykhi, M.H. Dehghani, S.H. Hendi, Phys. Rev. D 81, 084040 (2010). arXiv:0912.4199 [hep-th]

61. S.H. Hendi, A. Sheykhi, S. Panahiyan, B. Eslam Panah, Phys. Rev. D 92(6), 064028 (2015). arXiv:1509.08593 [hep-th]

62. M.H. Dehghani, S.H. Hendi, A. Sheykhi, H. Rastegar Sedehi, JCAP 0702, 020 (2007). arXiv:hep-th/0611288

63. M.K. Zangeneh, A. Sheykhi, M.H. Dehghani, Phys. Rev. D 91(4), 044035 (2015). arXiv:1505.01103 [gr-qc]

64. S.W. Hawking, D.N. Page, Commun. Math. Phys. 87, 577 (1983)

65. E. Witten, Adv. Theor. Math. Phys. 2, 505 (1998). arXiv:hep-th/9803131

66. S.S. Gubser, Phys. Rev. D 78, 065034 (2008). arXiv:0801.2977 [hep-th]
67. S.A. Hartnoll, C.P. Herzog, G.T. Horowitz, Phys. Rev. Lett. 101, 031601 (2008). arXiv:0803.3295 [hep-th]

68. J. Jing, Q. Pan, S. Chen, JHEP 1111, 045 (2011). arXiv:1106.5181 [hep-th]

69. Z. Zhao, Q. Pan, S. Chen, J. Jing, Nucl. Phys. B 871, 98 (2013). arXiv: 1212.6693

70. D. Roychowdhury, Phys. Rev. D 86, 106009 (2012). arXiv:1211.0904 [hep-th]

71. S. Gangopadhyay, Mod. Phys. Lett. A 29, 1450088 (2014). arXiv:1311.4416 [hep-th]

72. A. Dey, S. Mahapatra, T. Sarkar, JHEP 1406, 147 (2014). arXiv: 1404.2190 [hep-th]

73. A. Dey, S. Mahapatra, T. Sarkar, JHEP 1412, 135 (2014). arXiv:1409.5309 [hep-th]

74. C. Lai, Q. Pan, J. Jing, Y. Wang, Phys. Lett. B 749, 437 (2015) arXiv:1508.05926 [hep-th]

75. D. Ghorai, S. Gangopadhyay. Higher dimensional holographic superconductors in Born-Infeld electrodynamics with backreaction. Eur. Phys. J. C 76(3), 146 (2016). doi:10.1140/epjc/ s10052-016-4005-0. arXiv:1511.02444 [hep-th]

76. Y. Liu, Y. Gong, B. Wang, JHEP 1602, 116 (2016). arXiv:1505.03603 [hep-ph]

77. A. Sheykhi, H.R. Salahi, A. Montakhab. Analytical and Numerical Study of Gauss-Bonnet Holographic Superconductors with Power-Maxwell Field. JHEP 1604, 058 (2016). doi:10.1007/ JHEP04(2016)058. arXiv:1603.00075 [gr-qc]

78. R. Banerjee, S. Ghosh, D. Roychowdhury, Phys. Lett. B 696, 156 (2011). arXiv:1008.2644 [gr-qc]

79. R. Banerjee, S.K. Modak, S. Samanta, Phys. Rev. D 84, 064024 (2011). arXiv:1005.4832 [hep-th]

80. R. Banerjee, D. Roychowdhury, JHEP 1111 (2011) 004 arXiv: 1109.2433 [gr-qc]

81. R. Banerjee, S.K. Modak, D. Roychowdhury, JHEP 1210 (2012) 125. arXiv:1106.3877 [gr-qc]

82. S. Banerjee, S.K. Chakrabarti, S. Mukherji, B. Panda, Int. J. Mod. Phys. A 26, 3469 (2011). arXiv: 1012.3256 [hep-th]

83. O. Gurtug, S.H. Mazharimousavi, M. Halilsoy, Phys. Rev D 85, 104004 (2012). doi:10.1103/PhysRevD.85.104004. arXiv: 1010.2340 [gr-qc]

84. M. Hassaine, C. Martinez, Class. Quantum Gravity 25, 195023 (2008). doi:10.1088/0264-9381/25/19/195023. arXiv:0803.2946 [hep-th]

85. C. Martinez, A. Montecinos, Phase transitions in charged topological black holes dressed with a scalar hair. Phys. Rev. D 82, 127501 (2010). arXiv:1009.5681 [hep-th] 